\title{
Effect of an Oral Frailty Measures Program on Community-Dwelling Elderly People: A Cluster-Randomized Controlled Trial
}

\author{
Maki Shirobe ${ }^{a, b}$ Yutaka Watanabe ${ }^{c, d}$ Tomoki Tanaka $^{e}$ Hirohiko Hirano $^{d}$ \\ Takeshi Kikutani $^{f}$ Kazuko Nakajog ${ }^{9}$ Tetsuro Sato $^{\text {h }}$ Junichi Furuya ${ }^{\mathrm{i}}$ \\ Shunsuke Minakuchi ${ }^{\text {b }}$ Katsuya lijima ${ }^{e, j}$ \\ aThe Tokyo Metropolitan Support Center for Preventative Long-Term and Frail Elderly Care, Tokyo Metropolitan \\ Institute of Gerontology, Tokyo, Japan; ${ }^{\text {b}}$ Department of Gerodontology and Oral Rehabilitation, Graduate School \\ of Medical and Dental Sciences, Tokyo Medical and Dental University, Tokyo, Japan; 'Gerodontology, Department \\ of Oral Health Science, Faculty of Dental Medicine, Hokkaido University, Sapporo, Japan; ${ }^{\text {Research Team for }}$ \\ Promoting Independence and Mental Health, Tokyo Metropolitan Institute of Gerontology, Tokyo, Japan; Enstitute \\ of Gerontology, The University of Tokyo, Tokyo, Japan; 'Division of Clinical Oral Rehabilitation, The Nippon Dental \\ University Graduate School of Life Dentistry, Tokyo, Japan; ${ }^{9}$ Odawara Public Health and Welfare Center, Kanagawa \\ Prefectural Government, Odawara, Japan; hKanagawa Dental Association, Yokohama, Japan; 'Department of \\ Geriatric Dentistry, Showa University School of Dentistry, Tokyo, Japan; Institute for Future Initiatives, The \\ University of Tokyo, Tokyo, Japan
}

\section{Keywords}

Public health · Elderly people · Oral health · Oral frailty ·

Oral function

\begin{abstract}
Introduction: Oral frailty describes a trivial decline in the oral function and is considered to be related to frailty. Thus, effective management of oral frailty could prevent or ameliorate physical frailty and the need for care. However, there is a lack of consensus regarding specific interventions for oral frailty. In this cluster-randomized controlled trial, we investigated the effects of a newly developed oral frailty measures program mentored by dentists and dental hygienists for elderly people in a clinical setting. Methods: Of 3,296 participants included in a field survey, 219 who regularly visited dental clinics and met at least 3 of the following 6 criteria for oral frailty were considered eligible: $<20$ natural teeth, decreased chewing ability, decreased articulatory oral-motor
\end{abstract}

karger@karger.com www.karger.com/ger

Karger $\stackrel{\text { ' }}{=}$
(C) 2021 The Author(s)

Published by S. Karger AG, Basel

This is an Open Access article licensed under the Creative Common Attribution-NonCommercial-4.0 International License (CC BY-NC) (http://www.karger.com/Services/OpenAccessLicense), applicable to the online version of the article only. Usage and distribution for commercial purposes requires written permission. skills, decreased tongue pressure, and substantial subjective difficulties in eating and swallowing. After applying the inclusion and exclusion criteria, we studied 51 patients in the intervention group (14 men and 37 women; mean age, 78.6 years) and 32 patients in the control group ( 7 men and 25 women; mean age, 78.0 years). We implemented a 12-week oral frailty measures program only for the intervention group. The program included preparatory oral exercises, mouth-opening training, tongue pressure training, prosodic training, and masticatory training. Primary outcome measures were the chewing ability score, articulatory oral motor skill for /ta/, tongue pressure, subjective difficulty in eating tough foods, and subjective difficulty in swallowing. We compared baseline characteristics using the Mann-Whitney $U$ and $X^{2}$ tests for continuous and categorical variables, respectively. A repeated-measures two-way ANOVA was used to determine the efficacy of independent intervention variables, following the Wilcoxon signed-rank test. The groups (intervention/control) and time (baseline/week 12) were the 
independent variables. Oral frailty measures were the dependent variables. Results: Baseline characteristics and assessment results were similar between groups. We observed significant improvements in the intervention group in terms of articulatory oral motor skill for /ta/ and tongue pressure $(p<0.001)$. No improvements were observed in the control group. Discussion/Conclusion: Our results suggest that our oral frailty measures program effectively alleviates oral frailty. Future studies are needed to clarify the impact on preventing physical frailty and improving the nutritional status.

(c) 2021 The Author(s).

Published by S. Karger AG, Basel

\section{Introduction}

The association between oral function and physical frailty has been clarified in recent works [1-4]. In light of this association, the concept of oral frailty was conceptualized as an accumulated poor oral status, and its relation to frailty has been studied $[5,6]$. Oral frailty is defined as a series of phenomena and processes. In such cases, changes in various oral conditions associated with aging, combined with a decline in interest for oral health and a decline in physical and mental reserve capacity, could lead to an increase in oral weakness and impairments in mastication and swallowing and could impact on frailty $[7,8]$. The relationship between oral frailty and frailty was reported in a 4-year longitudinal study in elderly Japanese individuals [8]. They defined oral frailty as having 3 or more of the following conditions: $<20$ natural teeth, decreased chewing ability, decreased articulatory oral motor skills, decreased tongue pressure, and substantial subjective difficulties in eating and swallowing. They showed that oral frailty strongly predicted physical frailty, sarcopenia, the need for long-term care, and mortality. Thus, recognition of oral frailty may allow for early detection of physical frailty. In addition, the management of oral frailty may aid in preventing or ameliorating physical frailty and the need for care in elderly people. However, specific interventions that alleviate oral frailty have not yet been explored.

In Japan, there has been an increase in the average life expectancy and aging of society. This, in turn, has increased the nursing care needs, both in terms of the proportion of elderly people requiring nursing care and a prolongation of the period of care. Consequently, there has been a focus on developing resident-based services allowing individuals to remain active and independent. This eventually reduces their need for regular or continuous nursing care. The importance of oral health in pre- venting the need for nursing care is well-known [9-12]. However, the scarcity of evidence on remedial measures and the highly specialized nature of oral care hinder the incorporation of such programs into services for community-dwelling elderly people. Moreover, there are no such oral frailty measures programs in clinical practice.

To propose improvement measures that can be implemented across community-based services, we developed a new oral frailty measures program. Although the program does not include conventional dental treatment, direct training was introduced to address the decline in masticatory function. Consequently, the program is implemented by dentists or dental hygienists at dental clinics after determining the oral cavity condition and performing risk assessments for factors such as tooth fracture and prosthesis withdrawal. In this study, we investigated the effectiveness of our newly developed oral frailty measures program in alleviating oral frailty in elderly people.

\section{Materials and Methods}

\section{Study Design}

In this cluster-randomized controlled trial, we identified individuals with oral frailty from a 2016 survey conducted at 107 dental medical institutions at Kanagawa prefecture, Japan. Figure 1 is a study flowchart that summarizes the process of participant enrollment, allocation, follow-up, and analysis. This study was conducted following the CONSORT guidelines.

Among the 3,296 participants included in the field survey, we excluded those who attended health facilities other than dental clinics, did not regularly visit dental clinics, or required nursing care, from the trial. Those requiring some assistance in daily life were excluded from the study. Some individuals had cerebrovascular disorders and neurological and muscular diseases, but none of them had states that should be considered for the intervention program, and therefore, they were included. We included participants who met at least 3 of the 6 previously established criteria for oral frailty [8]. To obtain an adequate sample size, we approached the dental clinics with the largest number of patients with oral frailty. We selected 219 participants with oral frailty in 30 dental clinics, and randomly divided them into 2 groups based on their dental clinic. The intervention group comprised 122 patients (14 dental clinics), and the control group comprised 97 (16 dental clinics). Of these, 70 patients in the intervention group and 41 in the control group consented to the study; however, 19 in the intervention group and 9 in the control group discontinued their participation before the end of the trial. Clinic allocation was performed not by the interventionists or statistical analysts but by a third party using sealed-envelope randomization. The sheets of paper with the groups to be included in advance were placed in envelopes, and the papers were removed from the envelopes in order and assigned to the groups. The order was determined by randomly sorting the dental institution list using a random number function in Excel (Microsoft Corp, Redwood, WA, USA). 


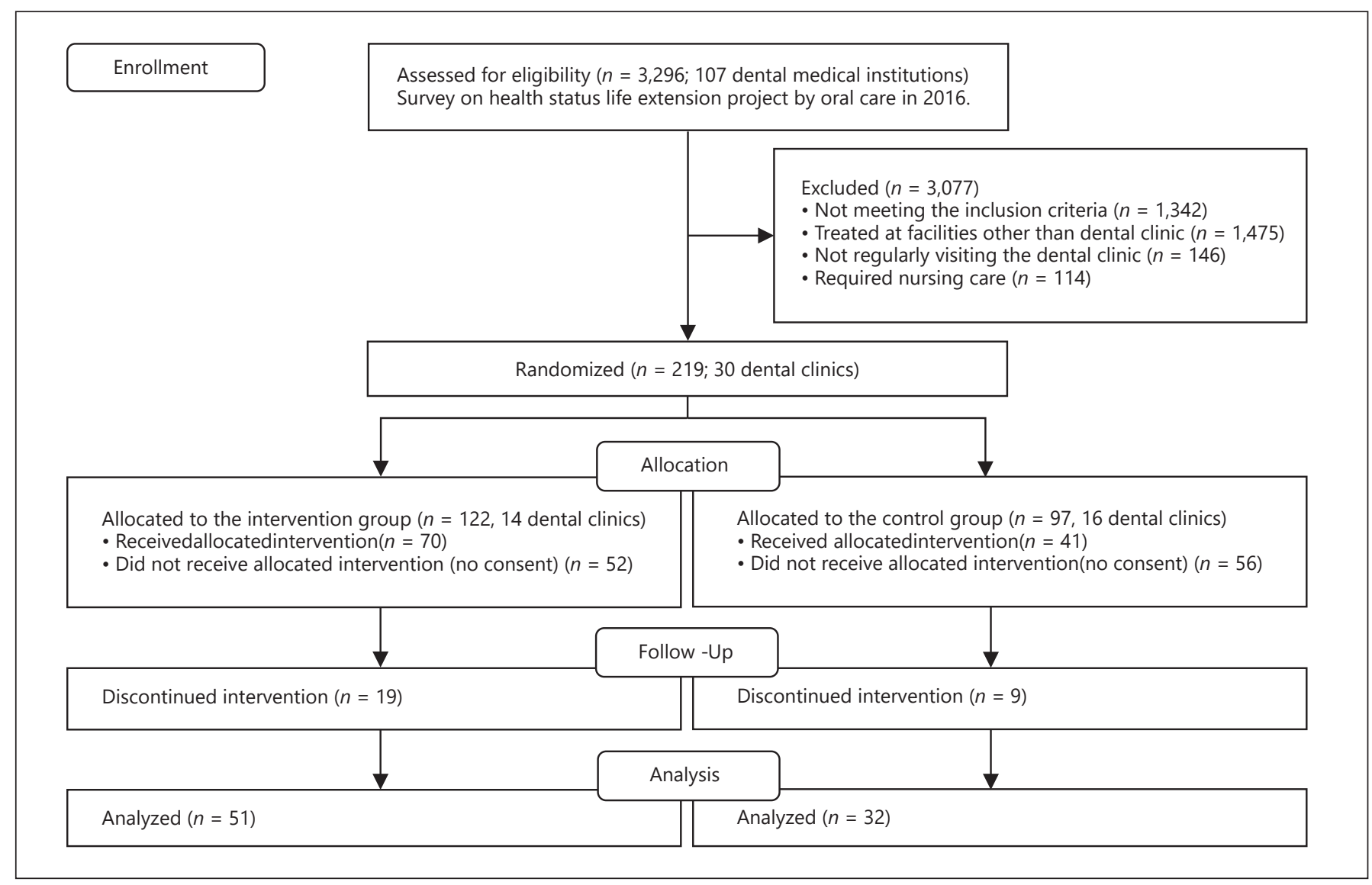

Fig. 1. Flowchart showing the enrollment process of the research participants with oral frailty. Adapted from the CONSORT 2010 flow diagram.

\section{Content of the Intervention}

We conducted a baseline assessment in both groups at the start of the study. This was followed by the implementation of a 12 -week program in the intervention group. The control group received no intervention during the same period. By the end of the 12 weeks, both groups were assessed again. Intervention group participants were asked to continue the program voluntarily for an additional 12 weeks to assess its sustained effects. Thereafter, we conducted a follow-up assessment. These participants did not receive any service between the 12- and 24-week assessments. For ethical reasons, after noting the advantage of the program at the 12-week assessment, a similar intervention program was implemented for the control group between weeks 12 and 24. Management of the initial 12 -week intervention and control groups was done at nonoverlapping dental clinics.

The intervention program was mentored by dentists or dental hygienists at their clinics at baseline and after 2, 4, and 8 weeks. They received prior training on the intervention techniques, regardless of their occupation. The interventionists explained the objectives and anticipated effects of the program at baseline. According to baseline assessment results, the program was tailored for each participant to address individual health parameters that suggested a decline in oral function. The program was implemented at regular intervals at home, and the participants had to docu- ment their activities on a calendar. The interventionists recorded the participants' status and provided guidance on the implementation of techniques during the follow-up visits. No dental treatment was offered at the clinics during the intervention period.

The program involved oral exercises, mouth-opening training, tongue-pressure training, prosodic training, and masticatory training. We prepared a program booklet describing these activities. All participants performed the oral exercises and one or more of the remaining 4 exercises when they met the respective criteria. Participants with a repetitive saliva swallowing test (RSST) score $<3$ in 30 s performed mouth-opening training. Participants with a gum test score $<3$ participated in masticatory training. Participants with a tongue pressure of $<30 \mathrm{kPa}$ performed tongue pressure training. Participants with oral diadochokinesis ([ODK]; /ta/) $<6.0$ times/s performed prosodic training. The cooperating dental clinics provided masticatory treatment to all participants who volunteered to receive such treatment.

Oral exercises included abdominal breathing and oral functional exercises $[13,14]$. Oral functional exercises consisted of short facial muscle gymnastics and a tongue gymnastics program that included the following 5 movements: goo, pah, guruguru, gokkun, and buh. In goo, an individual firmly closes the eyes and directs them downward, with the mouth firmly closed and the corners raised. In pah, the eyes are kept wide open and directed up- 
ward, with the mouth wide open. In guruguru, the tongue is moved from one side to the other, licking the inside of the lip, with the mouth closed. In gokkun, 3 movements are executed for swallowing the saliva accumulated inside the mouth. In buh, the tip of the tongue is pushed firmly out of the mouth, stretched, and held in position for $10 \mathrm{~s}$. These 5 movements were repeated three or more times each day.

In the mouth-opening exercise, the mouth is opened as wide as possible and held open for $10 \mathrm{~s}$, followed by $10 \mathrm{~s}$ of rest [15]. A set of 5 cycles was performed twice daily, in the morning and evening.

Tongue pressure training was performed using the Pecopanda (a tongue-strengthening training tool; JMS Inc., Tokyo, Japan) [16-18]. The tool is pushed upward with the tongue in a crushing motion, 5 times per set. Three sets were performed each day. The firmness of the device was set as $S$ for tongue pressure $\leq 10 \mathrm{kPa}, \mathrm{MS}$ for $11-15 \mathrm{kPa}$, and $\mathrm{M}$ for $16-30 \mathrm{kPa}$.

Prosodic training consisted of nonsense syllable chain training. A simple pattern of sounds produced using the lips $(/ \mathrm{ma} /, / \mathrm{ba} /)$, the tip of the tongue $(/ \mathrm{ta} /, / \mathrm{te} /)$, and the back of the tongue $(/ \mathrm{ka} /)$ was produced. Ten different nonsense words containing 3 sounds each were articulated. A complex pattern was produced by changing 2 of the 3 sounds simultaneously. The syllable chain was designed to maximize lip and tongue movement, with the requirement of rapid and clear pronunciation, and loud vocalizations. The training was performed 5 times, twice daily, before breakfast and lunch.

Masticatory training involved chewing gum twice daily, in the morning and at night. Rhythmic chewing for $2 \mathrm{~min}$ was followed by free chewing for $3 \mathrm{~min}$. The participants were instructed to close their lips and chew alternately and equally on both sides. In addition, they had to maintain an erect posture during this training.

\section{Outcomes}

Primary Outcome Measures

The presence or absence of oral frailty, the primary outcome, was evaluated based on 6 items. Although there are several methods for assessing oral frailty, in this study, we performed our assessment based on the method described by Tanaka et al. [8], which is a simple and feasible procedure for community-dwelling elderly people. If at least 3 of the following criteria were met, oral frailty was considered present: (1) the number of natural teeth $(<20)$; (2) the chewing ability score $(<3)$; (3) articulatory oral motor skill for "ta" ( $<6.0$ times/s); (4) tongue pressure ( $<30.0 \mathrm{kPa}) ;(5)$ subjective difficulty in eating tough foods (yes); and (6) subjective difficulty in swallowing (yes) [8]. For subjective difficulty in eating tough foods and swallowing, we used the questions from the Kihon checklist [19].

Secondary Outcome MeasuresCharacteristics of Participants in the Study

We examined the participants' data based on their sex, age, and medical history. These items were surveyed using a questionnaire. Moreover, we asked the participants regarding their previous history of dementia, cerebrovascular accident, diabetes, neuromuscular disease, hypertension, heart disease, and respiratory disease.

\section{Physical Function}

We investigated the Kihon checklist, body weight, body mass index (BMI), body fat percentage, muscle mass, basal metabolic rate, and call circumference. The Kihon checklist, a 25 -item ques- tionnaire, was used to predict frailty and disability [19]. The maximum score is 25 , and a higher score indicates greater frailty [20].

A body compositional meter (Inner Scan 50V BC-622; Tanita Corp., Tokyo, Japan) was used to measure patients' body weight, and to calculate their BMI, body fat percentage, muscle mass, and the basal metabolic rate. The calf circumference of the nondominant foot was measured at its thickest point, with the foot placed flat on the floor.

\section{Dental Items}

We investigated the numbers of natural and functional teeth, use of dentures, ODK test score, tongue pressure, RSST score, and chewing ability. The dental items were evaluated by dentists.

The number of natural teeth refers to teeth with intact crowns (excluding residual roots). The number of functional teeth was calculated as the number of prosthetic teeth that replaced missing natural teeth (including implants, pontics, and dentures) plus the number of natural teeth [1].

In the ODK test, participants repeatedly and rapidly pronounced/pa/,/ta/, and $/ \mathrm{ka} /$ monosyllables for $5 \mathrm{~s}$ each. The average number of repetitions per second was calculated [11].

Tongue pressure was measured using a JMS tongue pressure meter (TPM-01) [21, 22]. The balloon probe was adjusted automatically to a predetermined pressure and placed over the tongue. Thereafter, the tip of the tongue was pushed upward against the palate for approximately $7 \mathrm{~s}$ at maximum force. The maximum pressure value was recorded.

In the RSST, participants sat upright with their neck slightly flexed forward, and a finger was placed on the laryngeal prominence [23]. They were instructed to swallow saliva continuously. We measured the number of swallows perceived in $30 \mathrm{~s}$.

Masticatory performance was measured using the glucosensor GS-II (GC America, Inc., Alsip, IL, USA) [24]. Participants had to chew glucose-containing chewing gum for $20 \mathrm{~s}$, after which they gently rinsed their mouth using $10 \mathrm{~mL}$ of water. The chewing gum and water were expelled into a cup with a mesh filter. The GS-II was used to measure the glucose concentration of the filtered fluid.

Masticatory performance was also measured using a gum test, where patients chewed xylitol gum (Lotte Confectionery, Seoul, Korea) for $1 \mathrm{~min}$. We assessed the chewing function based on the change in color of the gum, using a five-level color chart [25-27].

\section{Sample Size}

We determined the sample size using GPower 3.1, as previously described [28]. Tanaka et al. [8] defined preoral frailty as an oral frailty score of 1-2. Considering that a decrease in the score of one point would affect the improvement of physical frailty, we set the effect size in the intervention group to one point. We assumed that the oral frailty score of the intervention group would decrease by an average of one point, while that of the control group would remain unchanged. A standard deviation of 1.5 points was used for both groups. Accordingly, we calculated that 37 participants were required for each group. Considering a dropout rate of $10 \%$, we aimed to recruit 41 participants for each group.

\section{Ethical Considerations}

The Institutional Review Board of the Faculty of Biodentistry, The Nippon Dental University (Tokyo, Japan), approved this study (NDU-T2017-10). It was registered at the University Hospi- 
Table 1. Participant characteristics at baseline survey

\begin{tabular}{|c|c|c|c|c|}
\hline & Overall $(n=83)$ & Intervention group $(n=51)$ & Control group $(n=32)$ & $p$ value \\
\hline Sex, female & $62(74.7)$ & $37(72.5)$ & $25(78.1)$ & 0.614 \\
\hline Age, years & $78.0(67.0,90.0)$ & $79.0(68.0,90.0)$ & $78.0(67.0,89.0)$ & 0.715 \\
\hline \multicolumn{5}{|l|}{ Medical history } \\
\hline Cerebrovascular accident & $4(4.8)$ & $4(7.8)$ & $0(0.0)$ & 0.156 \\
\hline Dementia & $3(3.6)$ & $2(3.9)$ & $1(3.1)$ & 1.000 \\
\hline Diabetes mellitus & $3(3.6)$ & $3(5.9)$ & $0(0.0)$ & 0.281 \\
\hline Hypertension & $32(38.6)$ & $20(39.2)$ & $12(37.5)$ & 1.000 \\
\hline Heart disease & $7(8.4)$ & $4(7.8)$ & $3(9.3)$ & 1.000 \\
\hline Respiratory diseases & $6(7.2)$ & $5(9.8)$ & $1(3.1)$ & 0.398 \\
\hline $\mathrm{KCL}$ & $5.0(0.0,17.0)$ & $4.5(0.0,14.0)$ & $5.0(0.0,17.0)$ & 0.320 \\
\hline Height, cm & $150.4(134.2,180.0)$ & $150.0(136.0,170.0)$ & $152.0(134.2,180.0)$ & 0.732 \\
\hline Weight, kg & $51.8(35.2,79.8)$ & $51.3(37.2,79.8)$ & $51.9(35.2,75.4)$ & 0.722 \\
\hline $\mathrm{CC}, \mathrm{cm}$ & $33.0(24.0,40.0)$ & $33.0(26.0,40.0)$ & $33.0(24.0,37.0)$ & 0.981 \\
\hline Remaining natural teeth, $n$ & $13.0(0.0,30.0)$ & $14.0(0.0,30.0)$ & $12.0(0.0,25.0)$ & 0.101 \\
\hline Functional teeth, $n$ & $28.0(7.0,30.0)$ & $28.0(7.0,29.0)$ & $28.0(12.0,30.0)$ & 0.364 \\
\hline ODK/pa/, times/s & $5.0(3.0,6.8)$ & $4.8(3.4,6.6)$ & $5.2(3.0,6.8)$ & 0.112 \\
\hline $\mathrm{ODK} / \mathrm{ta} /$, times/s & $5.0(2.8,7.0)$ & $5.0(3.2,6.6)$ & $5.1(2.8,7.0)$ & 0.649 \\
\hline ODK/ka/, times/s & $4.8(2.4,7.6)$ & $4.8(3.4,6.4)$ & $5.1(2.4,7.6)$ & 0.161 \\
\hline Tongue pressure, $\mathrm{kPa}$ & $27.4(2.6,47.1)$ & $24.7(2.6,47.1)$ & $28.4(15.4,42.9)$ & 0.163 \\
\hline RSST, times & $4.0(1.0,8.0)$ & $4.0(1.0,7.0)$ & $4.0(1.0,8.0)$ & 0.712 \\
\hline Glucosensor, mg/dL & $139.0(13.0,254.0)$ & $125.0(13.0,218.0)$ & $148.0(21.5,254.0)$ & 0.189 \\
\hline Gum test score & $4.0(1.0,5.0)$ & $4.0(1.0,5.0)$ & $4.0(2.0,5.0)$ & 0.259 \\
\hline OF score & $3.0(1.0,5.0)$ & $3.0(1.0,5.0)$ & $3.0(1.0,5.0)$ & 0.938 \\
\hline
\end{tabular}

Data are presented as $n$ (\%) or the median (min., max.). $p$ values were calculated using the Mann-Whitney $\mathrm{U}$ test or a $\chi^{2}$ test. KCL, Kihon checklist score; BFP, body fat percentage; BMI, body mass index; BMR, basal metabolic rate; CC, calf circumference; No., number of; ODK, oral diadochokinesis; RSST, repetitive saliva swallowing test; OF, oral frailty; Min, minimum; Max, maximum.

tal Medical Information Network (UMIN000029358). The participants were enrolled after we obtained their signed informed consent. This ensured that they were aware of the purpose, methods, and other aspects of the study. They were informed that they were free to withdraw their consent at any time without any detrimental consequences.

\section{Statistical Analyses}

We compared baseline characteristics using the Mann-Whitney $U$ and $\chi^{2}$ tests for continuous and categorical variables, respectively. A repeated-measures two-way ANOVA was used to determine the efficacy of independent intervention variables, following the Wilcoxon signed-rank test. The groups (intervention/ control) and time (baseline/week 12) were the independent variables. Oral frailty measures were the dependent variables. We used a repeated-measures two-way ANOVA to examine program continuation effects. The Bonferroni method was used for post hoc testing. We performed all statistical analyses using IBM SPSS Statistics for Windows, version 24.0 (IBM Corp., Armonk, NY, USA). Differences with a $p$ value $<0.05$ were considered statistically significant.

Effect of an Oral Frailty Measures Program

\section{Results}

\section{Participants}

After excluding participants who withdrew from the study due to personal or family reasons, 51 patients were included in the intervention group (14 men and 37 women; mean \pm standard deviation age, $78.6 \pm 5.8$ years) and 32 in the control group ( 7 men and 25 women; $78.0 \pm 6.8$ years) (Fig. 1).

\section{Baseline Assessment}

The groups were similar in their baseline characteristics and assessment results (Table 1).

\section{Intervention Effect}

We compared the assessment results between the 2 groups before and after the intervention (Table 2). In the intervention group, we observed a significant increase in 


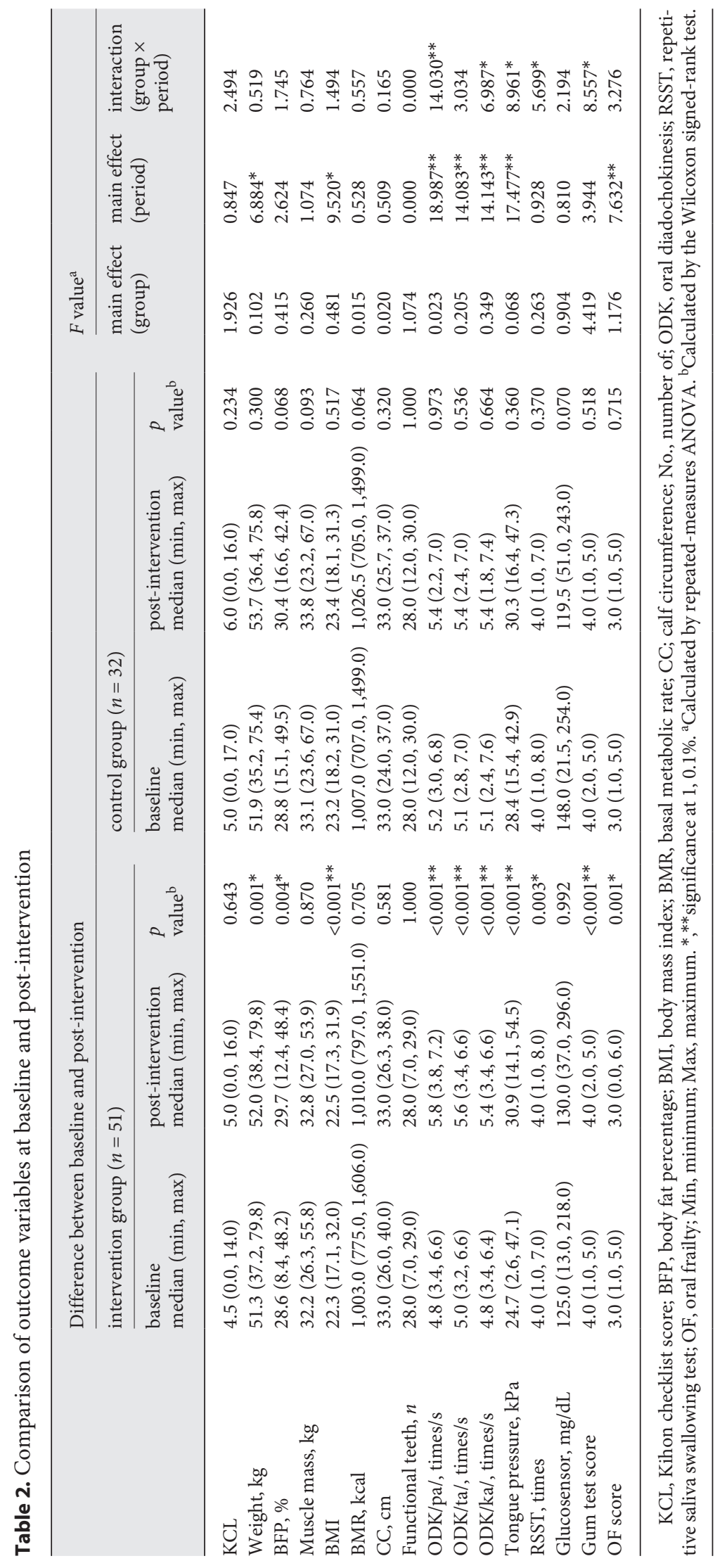


Table 3. Continuation effect of the intervention group

\begin{tabular}{|c|c|c|c|c|c|}
\hline Weight, kg & $51.3(37.2,79.8)$ & $52.0(38.4,79.8)$ & $51.8(39.4,80.2)$ & 5.94 & $0.011^{*}$ \\
\hline $\mathrm{BFP}, \%$ & $28.6(8.4,48.2)^{\mathrm{a}}$ & $29.7(12.4,48.4)$ & $29.5(10.2,44.3)^{\mathrm{a}}$ & 7.37 & $0.002^{* *}$ \\
\hline Muscle mass, kg & $32.2(26.3,55.8)$ & $32.8(27.0,53.9)$ & $32.8(27.4,53.0)$ & 0.20 & 0.771 \\
\hline $\mathrm{CC}, \mathrm{cm}$ & $33.0(26.0,40.0)$ & $33.0(26.3,38.0)$ & $33.0(26.3,38.5)$ & 1.26 & 0.276 \\
\hline Functional teeth, $n$ & $28.0(7.0,29.0)$ & $28.0(7.0,29.0)$ & $28.0(7.0,29.0)$ & 1.21 & 0.278 \\
\hline ODK/pa/, times/s & $4.8(3.4,6.6)^{\mathrm{a}}$ & $5.8(3.8,7.2)^{\mathrm{b}}$ & $5.8(4.2,7.4)^{\mathrm{a}, \mathrm{b}}$ & 52.73 & $<0.001^{* *}$ \\
\hline ODK/ta/, times/s & $5.0(3.2,6.6)^{\mathrm{a}}$ & $5.6(3.4,6.6)^{\mathrm{b}}$ & $5.8(4.0,7.2)^{\mathrm{a}, \mathrm{b}}$ & 37.88 & $<0.001^{* *}$ \\
\hline ODK/ka/, times/s & $4.8(3.4,6.4)^{\mathrm{a}}$ & $5.4(3.4,6.6)^{\mathrm{b}}$ & $5.4(3.8,6.6)^{\mathrm{a}, \mathrm{b}}$ & 40.14 & $<0.001^{* *}$ \\
\hline Tongue pressure, $\mathrm{kPa}$ & $24.7(2.6,47.1)^{\mathrm{a}}$ & $30.9(14.1,54.5)$ & $30.9(16.2,49.5)^{\mathrm{a}}$ & 26.59 & $<0.001^{* *}$ \\
\hline OF score & $3.0(1.0,5.0)^{\mathrm{a}}$ & $3.0(0.0,6.0)^{\mathrm{b}}$ & $2.0(0.0,6.0)^{\mathrm{a}, \mathrm{b}}$ & 16.88 & $<0.001^{* *}$ \\
\hline
\end{tabular}

KCL, Kihon checklist score; BFP, body fat percentage; BMI, body mass index; BMR, basal metabolic rate; CC, calf circumference; No., number of; ODK, oral diadochokinesis; RSST, repetitive saliva swallowing test; OF, oral frailty; Min, minimum; Max, maximum. *** significance at 5, 0.1\%. a Significant difference was detected between baseline and follow-up by the Bonferroni method. bignificant difference was detected between post-intervention and follow-up by the Bonferroni method.

body weight, body fat percentage, BMI, ODK (/pa/, /ta/, and $/ \mathrm{ka} /$ ), tongue pressure, RSST, the gum test score, and the oral frailty score $(p<0.05)$. In addition, there was a significant interaction between groups and time for ODK (/pa/), ODK (/ka/), tongue pressure, and RSST and gum test results (Table 2).

\section{Continuation Effect}

Table 3 summarizes the effects of voluntary program continuation by participants in the intervention group. Particularly, the items for which there were significant differences between the post-intervention survey and the 24-week follow-up period, as continuation effects, were ODK (/pa/, /ta/, and /ka/), glucosensor, RSST, the gum test, and oral frailty scores $(p<0.05)$.

\section{Discussion}

In this study, we observed a significant increase in oral function in the intervention group. This was concomitant with a substantial decrease in the number of items indicative of oral frailty. In contrast, there was no improvement in oral function in the control group. This suggests that the oral frailty measures program was responsible for alleviating participants' oral frailty. Previous studies, conducted among community-dwelling elderly people, have reported on the efficacy of an educational intervention based on oral gymnastics and oral health behaviors, including exercises of the tongue, lips, and cheeks. Weekly interventions were implemented for $60-90 \mathrm{~min}$ at a time, spanning 12 weeks $[11,14,29]$. However, we are not aware of similar studies in which long-term effects or detailed examinations were reported.

Participants who voluntarily continued with the program after the intervention period exhibited substantial improvements in various outcomes relative to values documented at the end of the 12-week intervention. Routine use of the oral cavity in conversation and eating can prevent a minor decline in oral function. We have demonstrated even better results by adapting our program to everyday life by incorporating elements such as oral cleaning and chewing. Individuals can consciously engage in consumption of tough food, elaborate chewing, repeated swallowing, and use of large mouth movements while speaking. Thus, beneficial effects can be achieved even without direct professional intervention.

The program's efficacy persisted for at least 3 months after implementation of the intervention because participants continued to perform the exercises, despite not re- 
ceiving further professional assistance. Monitoring of oral health at least once every 12 weeks is expected to facilitate the identification of any decline in oral function. For successful program execution, cooperation with dental clinics and dental hospitals for emergency management of accidents and deterioration of oral conditions is imperative. However, the program can also be implemented through community-based services. Assessments, however, would need to be performed by dental professionals as specialized equipment is required. If the previous conditions are met, such a program would likely lead to sustained effects.

To consider the role of oral health in determining the need for nursing care [8], we evaluated patients' daily life activities and physical exercise, following the Kihon checklist [19]. However, we observed no changes in these parameters in either group. Nonetheless, a statistically significant increase in body weight, body fat percentage, and BMI was observed in the intervention group postintervention. An increase in the body fat percentage and BMI should be considered in terms of the impact on obesity. However, the mean values in both groups were within the Japanese reference values at all assessment time points. In addition, there was little impact on the body. The increase in weight, BMI, and percentage of body fat is thought to be due to an increase in the intake of energy and fat. Since we did not evaluate the actual diet in this study, this is only a presumption; nevertheless, several associations between oral function and energy intake and nutrients have been reported [30-32]. Motokawa et al. [32] reported that the intake of nutrients such as energy and fat was significantly lower in the group with compromised masticatory function than the group with good masticatory function, and there were also lower intake levels in many other food groups. There are also several reports on oral function and dietary variety [33, 34]. Kwon et al. [33] reported that one of the factors influencing a decrease in dietary variety is the decreased chewing ability. Therefore, it was inferred that the increase in body weight, body fat percentage, and BMI was due to the improvement in oral frailty, which resulted in improved masticatory function and increased energy and fat intake.

A considerable dropout rate during the intervention period was a major limitation of this study. We expected a lower dropout rate because the participants regularly visited the dental clinics and had already established a good rapport with their respective dentist and dental hygienist. Moreover, they appeared highly motivated to participate in this study. The physical condition of the participants and/or their family members was the main reason for the dropouts. Coaching for the intervention was implemented by previously trained dentists or dental hygienists, using the program booklet. They carefully assessed the participants during the intervention period and provided regular feedback to keep participants motivated during the program. Because we conducted a cluster-randomized controlled trial to explore the differences in the intervention content, the risk of bias was low. Additionally, although BMI was used to assess the nutritional status in this study, the association between oral frailty and nutrition may have become more apparent with other nutritional indicators, such as energy quantity and the Mini Nutritional Assessment-Short Form [35], and assessments of diet quality, such as the texture of food and the dietary variety score [36]. Future studies should follow the participants' progress in alleviating oral frailty over a long period and determine the impact on the prevention of physical frailty and improvement in nutritional status, including the addition of evaluation measures. Moreover, this study assessed those who did not require nursing care but had a history of dementia, neuromuscular diseases, respiratory diseases, and others. The study did not include those who required special consideration for implementing the intervention.

The findings of this cluster-randomized controlled trial suggest that oral frailty measures program implementation at dental clinics alleviates oral frailty in elderly people. Moreover, a continued effect was observed for at least 3 months after completion of the program because participants continued to perform the exercises, despite not receiving further professional assistance. The program might play an important role in the prevention and improvement of oral frailty in the elderly.

To develop this program, it should not be limited to a dental clinic, but needs to be implemented continuously in the community where the elderly individuals live. For this purpose, it is necessary to implement the program at home and places, such as residents' voluntary groups, and to develop a backup system at dental institutions. In addition, it is also important to collaborate with nutritionists, rehabilitation professionals, and physicians in consideration of the fact that the activities are connected with frailty. It is expected that the elderly will take the initiative and be supported by multiple professions to effectively improve oral frailty.

\section{Acknowledgements}

We are grateful to the staff of the dental clinics in Kanagawa prefecture for participating in this study. We are also grateful to the following project team members: Katsushi Tamaki, Haruka Tohara, Shouji Hironaka, Tatsuo Yamamoto, and Takuo Ishii.
Shirobe et al. 


\section{Statement of Ethics}

The Institutional Review Board of the Faculty of Biodentistry, The Nippon Dental University, approved this study (NDUT2017-10). The participants were considered eligible only if they had signed the informed consent form, which explained the purpose, methods, and other measures of the study. They were free to withdraw their consent at any time without detrimental consequences. This study followed the principles of the Declaration of Helsinki (updated version 2008).

\section{Conflict of Interest Statement}

The authors have no conflicts of interest to declare.

\section{Funding Sources}

This study was supported by the Healthy Life Extension by Oral Cavity Care Project at Kanagawa prefecture, 2017. The funding source had no role in the preparation of the data or the manuscript.

\section{Author Contributions}

Shirobe M. and Watanabe Y.: contributed to the conception and design of the study, analysis and interpretation of the results, and drafting and critically revising the manuscript. Tanaka T. and Kikutani T.: contributed to the conception and design of the study, interpretation of the results, and have critically revised the manuscript. Hirano H.: contributed to the conception and design of the study, analysis and interpretation of the results, and has critically revised the manuscript. Nakajo K. and Sato T.: contributed to the conception and design of the study, acquisition of the data, interpretation of the results, and have critically revised the manuscript. Furuya J. and Minakuchi S.: contributed to the conception of the study, interpretation of the results, and have critically revised the manuscript. Iijima K.: contributed to the conception and design of the study, acquisition and analysis of the data, interpretation of the results, and drafting and critically revising the manuscript.

\section{References}

1 Watanabe $\mathrm{Y}$, Hirano $\mathrm{H}$, Arai $\mathrm{H}$, Morishita $\mathrm{S}$, Ohara Y, Edahiro A, et al. Relationship between frailty and oral function in communitydwelling elderly adults. J Am Geriatr Soc. 2017 Jan;65(1):66-76.

2 Iwasaki M, Kimura Y, Sasiwongsaroj K, Kettratad-Pruksapong M, Suksudaj S, Ishimoto Y, et al. Association between objectively measured chewing ability and frailty: a crosssectional study in central Thailand. Geriatr Gerontol Int. 2018 Jun;18(6):860-6.

3 Tôrres LH, Tellez M, Hilgert JB, Hugo FN, de Sousa MD, Ismail AI. Frailty, frailty components, and oral health: a systematic review. J Am Geriatr Soc. 2015 Dec;63(12):2555-62.

4 Iwasaki M, Yoshihara A, Sato N, Sato M, Minagawa K, Shimada M, et al. A 5-year longitudinal study of association of maximum bite force with development of frailty in community-dwelling older adults. J Oral Rehabil. 2018 Jan;45(1): 17-24.

5 Payne M, Morley JE. Editorial: dysphagia, dementia and frailty. J Nutr Health Aging. 2018 Apr;22(5):562-5.

6 Hihara T, Goto T, Ichikawa T. Investigating eating behaviors and symptoms of oral frailty using questionnaires. Dent J. 2019 Jun;7(3): 66.

7 Hirano H. Management of oral-frailty in elderly. Jpn J Gerodontol. 2017 Mar;31(4):4004.

8 Tanaka T, Takahashi K, Hirano H, Kikutani $\mathrm{T}$, Watanabe Y, Ohara Y, et al. Oral frailty as a risk factor for physical frailty and mortality in community-dwelling elderly. J Gerontol A Biol Sci Med Sci. 2018 Nov;73(12):1661-7.
9 Haumschild MS, Haumschild RJ. The importance of oral health in long-term care. J Am Med Dir Assoc. 2009 Nov;10(9):667-71.

10 Morishita S, Watanabe Y, Hirano H, Edahiro A, Ohara Y, Shirobe M, et al. A long-term intervention research for the effect of nutrition and oral function improvement service at the day care facilities. J Jpn Soc Dent Hyg. 2017 Aug;12(1):36-46.

11 Sakayori T, Maki Y, Hirata S, Okada M, Ishii T. Evaluation of a Japanese "prevention of long-term care" project for the improvement in oral function in the high-risk elderly. Geriatr Gerontol Int. 2013 Apr;13(2):451-7.

12 Rapp L, Sourdet S, Vellas B, Lacoste-Ferré MH. Oral health and the frail elderly. J Frailty Aging. 2017 Jan;6(3):154-60.

13 Kikutani T, Enomoto R, Tamura F, Oyaizu K, Suzuki A, Inaba S. Effects of oral functional training for nutritional improvement in Japanese older people requiring long-term care. Gerodontology. 2006 Jun;23(2):93-8.

14 Ohara Y, Yoshida N, Kono Y, Hirano H, Yoshida $\mathrm{H}$, Mataki S, et al. Effectiveness of an oral health educational program on community-dwelling older people with xerostomia. Geriatr Gerontol Int. 2015 Apr;15(4):481-9.

15 Wada S, Tohara H, Iida T, Inoue M, Sato M, Ueda K. Jaw-opening exercise for insufficient opening of upper esophageal sphincter. Arch Phys Med Rehabil. 2012 Nov;93(11):1995-9.

16 Otaki H, Sato S, Okita K, Okamoto T. Quantitative evaluation of the suprahyoid muscle activity using the tongue training instrument "Peko-panda. Jpn J Speech Lang Hear Res. 2017 Jun;14(2):134-8.
17 Yano J, Yamamoto-Shimizu S, Yokoyama T, Kumakura I, Hanayama K, Tsubahara A. Effects of tongue-strengthening exercise on the geniohyoid muscle in young healthy adults. Dysphagia. 2020 Feb;35(1): 110-6.

18 Robbins J, Kays SA, Gangnon RE, Hind JA, Hewitt AL, Gentry LR, et al. The effects of lingual exercise in stroke patients with dysphagia. Arch Phys Med Rehabil. 2007 Feb;88(2): $150-8$.

19 Arai H, Satake S. English translation of the Kihon checklist. Geriatr Gerontol Int. 2015 Apr; 15(4):518-9.

20 Satake S, Senda K, Hong YJ, Miura H, Endo $\mathrm{H}$, Sakurai T, et al. Validity of the Kihon Checklist for assessing frailty status. Geriatr Gerontol Int. 2016 Jun;16(6):709-15.

21 Machida N, Tohara H, Hara K, Kumakura A, Wakasugi Y, Nakane A, et al. Effects of aging and sarcopenia on tongue pressure and jawopening force. Geriatr Gerontol Int. $2017 \mathrm{Feb}$; 17(2):295-301.

22 Tsuga K, Yoshikawa M, Oue H, Okazaki Y, Tsuchioka H, Maruyama M, et al. Maximal voluntary tongue pressure is decreased in Japanese frail elderly persons. Gerodontology. 2012 Jun;29(2):e1078-85.

23 Tamura F, Mizukami M, Ayano R, Mukai Y. Analysis of feeding function and jaw stability in bedridden elderly. Dysphagia. 2002 Jul; 17(3):235-41.

24 Uesugi $H$, Shiga H. Relationship between masticatory performance using a gummy jelly and masticatory movement. J Prosthodont Res. 2017 Oct;61(4):419-25. 
25 Hama Y, Kanazawa M, Minakuchi S, Uchida T, Sasaki Y. Properties of a color-changeable chewing gum used to evaluate masticatory performance. J Prosthodont Res. 2014 Apr; 58(2):102-6.

26 Horie T, Kanazawa M, Komagamine Y, Hama Y, Minakuchi S. Association between near occlusal contact areas and mixing ability. J Oral Rehabil. 2014 Nov;41(11):829-35.

27 Komagamine Y, Kanazawa M, Kaiba Y, Sato Y, Minakuchi S, Sasaki Y. Association between self-assessment of complete dentures and oral health-related quality of life. J Oral Rehabil. 2012 Nov;39(11):847-57.

28 Faul F, Erdfelder E, Buchner A, Lang AG. Statistical power analyses using $G^{*}$ Power 3.1: tests for correlation and regression analyses. Behav Res Methods. 2009 Nov;41(4):114960.

29 Wang TF, Huang CM, Chou C, Yu S. Effect of oral health education programs for caregivers on oral hygiene of the elderly: a systemic review and meta-analysis. Int J Nurs Stud. 2015 Jun;52(6):1090-6.

30 Mann T, Heuberger R, Wong H. The association between chewing and swallowing difficulties and nutritional status in older adults. Aust Dent J. 2013 Jun;58(2):200-6.

31 Iwasaki M, Taylor GW, Manz MC, Yoshihara A, Sato M, Muramatsu K, et al. Oral health status: relationship to nutrient and food intake among 80-year-old Japanese adults. Community Dent Oral Epidemiol. 2014 Oct; 42(5):441-50.

32 Motokawa K, Mikami Y, Shirobe M, Edahiro A, Ohara Y, Iwasaki M, et al. Relationship between chewing ability and nutritional status in Japanese older adults: a cross-sectional study. Int J Environ Res Public Health. 2021 Jan;18(3):1216.

33 Kwon J, Suzuki T, Kumagai S, Shinkai S, Yukawa H. Risk factors for dietary variety de- cline among Japanese elderly in a rural community: a 8-year follow-up study from TMIGLISA. Eur J Clin Nutr. 2006 Mar;60(3): 305-11.

34 Kagawa R, Ikebe K, Inomata C, Okada T, Takeshita H, Kurushima Y, et al. Effect of dental status and masticatory ability on decreased frequency of fruit and vegetable intake in elderly Japanese subjects. Int J Prosthodont. 2012 Jul-Aug;25(4):368-75.

35 Ulger Z, Halil M, Cankurtaran M, Yavuz BB, Yesil Y, Kuyumcu ME, et al. Malnutrition in Turkish nursing homes: a correlate of shortterm mortality. J Nutr Health Aging. 2013 Apr;17(4):305-9.

36 Kumagai S, Watanabe S, Shibata H, Amano H, Fujiwara Y, Shinkai S, et al. [Effects of dietary variety on declines in high-level functional capacity in elderly people living in a community]. Nihon Koshu Eisei Zasshi. 2003 Dec;50(12):1117-24 